\title{
Metformin activates AMP-activated protein kinase in primary human hepatocytes by decreasing cellular energy status
}

\author{
X. Stephenne - M. Foretz $\cdot$ N. Taleux • \\ G. C. van der Zon • E. Sokal • L. Hue • B. Viollet • \\ B. Guigas
}

Received: 30 July 2011 /Accepted: 18 August 2011 /Published online: 23 September 2011

(C) The Author(s) 2011. This article is published with open access at Springerlink.com

\begin{abstract}
Aim/hypothesis The glucose-lowering drug metformin has been shown to activate hepatic AMP-activated protein kinase (AMPK), a master kinase regulating cellular energy homeostasis. However, the underlying mechanisms remain controversial and have never been investigated in primary human hepatocytes.

Methods Hepatocytes isolated from rat, mouse and human livers were treated with various concentrations of metformin. Isoform-specific AMPK $\alpha$ abundance and activity, as well as intracellular adenine nucleotide levels and mitochondrial oxygen consumption rates were determined at different time points.

Results Metformin dose- and time-dependently increased AMPK activity in rat and human hepatocytes, an effect associated with a significant rise in cellular AMP:ATP ratio. Surprisingly, we found that AMPK $\alpha 2$ activity was unde-
\end{abstract}

Electronic supplementary material The online version of this article (doi:10.1007/s00125-011-2311-5) contains peer-reviewed but unedited supplementary material, which is available to authorised users.

X. Stephenne $\cdot$ E. Sokal

Laboratory of Paediatric Hepatology and Cell Therapy,

Université catholique de Louvain and Cliniques St Luc,

Brussels, Belgium

M. Foretz $\cdot$ B. Viollet

Inserm, U1016, Institut Cochin,

Paris, France

M. Foretz $\cdot$ B. Viollet

CNRS, UMR8104,

Paris, France

M. Foretz $\cdot$ B. Viollet

Université Paris Descartes,

Paris, France tectable in human compared with rat hepatocytes, while AMPK $\alpha 1$ activities were comparable. Accordingly, metformin only increased AMPK $\alpha 1$ activity in human hepatocytes, although both AMPK $\alpha$ isoforms were activated in rat hepatocytes. Analysis of mRNA expression and protein levels confirmed that only AMPK $\alpha 1$ is present in human hepatocytes; it also showed that the distribution of $\beta$ and $\gamma$ regulatory subunits differed between species. Finally, we demonstrated that the increase in AMP:ATP ratio in hepatocytes from liver-specific Ampko1/2 (also known as Prkaa1/2) knockout mice and humans is due to a similar and specific inhibition of the mitochondrial respiratorychain complex 1 by metformin.

Conclusions/interpretation Activation of hepatic AMPK by metformin results from a decrease in cellular energy status owing to metformin's AMPK-independent inhibition of the mitochondrial respiratory-chain complex 1 . The unique

N. Taleux $\cdot$ L. Hue $\cdot$ B. Guigas

Hormone and Metabolic Research Unit,

Université catholique de Louvain and de Duve Institute,

Brussels, Belgium

N. Taleux

Bioénergétique Fondamentale et Appliquée Inserm-U884,

Université J. Fourier,

Grenoble, France

G. C. van der Zon • B. Guigas ( $₫)$

Department of Molecular Cell Biology,

Leiden University Medical Center,

Postzone S1-P, Postbus 9600,

2300 RC Leiden, the Netherlands

e-mail: b.guigas@lumc.nl 
profile of AMPK subunits found in human hepatocytes should be considered when developing new pharmacological agents to target the kinase.

Keywords AMP:ATP ratio - AMPK · Hepatocytes . Human $\cdot$ Metformin $\cdot$ Mitochondria $\cdot$ Respiratory-chain complex 1

$\begin{array}{ll}\text { Abbreviations } \\ \text { ACC } & \text { Acetyl-CoA carboxylase } \\ \text { AMPK } & \text { AMP-activated protein kinase } \\ \text { AMPKK } & \text { AMPK kinase } \\ \mathrm{DNP} & \text { 2,4-Dinitrophenol } \\ \mathrm{JO}_{2} & \text { Mitochondrial oxygen consumption rate } \\ \mathrm{OCT}_{1} & \text { Organic cation transporter 1 } \\ \text { OXPHOS } & \text { Oxidative phosphorylation }\end{array}$

\section{Introduction}

Metformin is one of the most widely used glucose-lowering agents to treat type 2 diabetes [1] and is now recommended as the first-line drug therapy by a recent joint consensus statement [2]. This unique survivor of the biguanides family is prescribed for its effective anti-hyperglycaemic action, achieving a potent reduction of hepatic glucose production through inhibition of gluconeogenesis [3]. An increase in peripheral glucose uptake by the drug has also been reported, although the extent and significance of this effect in humans is still being discussed [3]. Metformin also exerts beneficial effects on circulating lipids and exhibits cardio-protective features in obese patients treated with the drug compared with conventional hypoglycaemic agents [4]. More recently, epidemiological studies have shown a decrease in cancer incidence in metformin-treated patients, suggesting a new potential application of the drug as an anti-cancer agent [5]. However, although prescribed since the end of the 1950s and despite extensive effort during the last years, the precise molecular (or biochemical) mechanism or mechanisms of action of metformin remain as yet incompletely understood.

At the beginning of the millennium, a remarkable study by Zhou et al. provided a new mechanistic insight by showing for the first time that metformin activates the AMP-activated protein kinase (AMPK) in hepatocytes; however, they did not provide an underlying mechanism for this activation [6]. More recently, Shaw et al. reported that activation of the hepatic LKB1/AMPK axis in mice could be involved in the inhibitory effect of metformin on gluconeogenesis, highlighting the putative role of these kinases in the therapeutic action of the drug [7]. AMPK is a well conserved serine/threonine-protein kinase, which acts as a cellular energy and nutrient sensor, and plays a crucial role in the regulation of metabolic pathways $[8,9]$. AMPK consists of a heterotrimeric complex containing a catalytic subunit $\alpha$ and two regulatory $\beta$ and $\gamma$ subunits. Each subunit has several isoforms $(\alpha 1, \alpha 2, \beta 1, \beta 2, \gamma 1, \gamma 2, \gamma 3)$, which are encoded by distinct genes, giving multiple combinations of holoenzyme with different tissue distribution and cellular localisation $[8,9]$. The $\alpha$ subunit contains a threonine residue (Thr 172) whose phosphorylation by upstream kinases (AMPK kinase [AMPKK]), such as the serine/threonine-protein kinase LKB1 or calmodulindependent protein kinase kinase II $\beta$ (CaMKKII $\beta)$, is sufficient and necessary for AMPK activation to occur. The $\beta$ subunit acts as a scaffold to which the two other subunits are bound, and also allows AMPK to sense energy reserves in the form of glycogen $[8,9]$. Binding of AMP to the $\gamma$ subunit activates AMPK via a complex mechanism involving direct allosteric activation, phosphorylation on Thr172 by AMPKK and inhibition of dephosphorylation of this residue by specific protein phosphatases that remain to be identified $[8,9]$. Thus, any increase in the intracellular AMP:ATP ratio activates AMPK, which results, through phosphorylation of various downstream targets, in concomitant inhibition of energy-consuming processes and stimulation of ATP-generating pathways in order to restore energy balance $[8,9]$. Finally, it has recently been shown that ADP, and therefore the ADP:ATP ratio, could also play a regulatory role in AMPK activities by binding to selective Bateman domains on the $\gamma$ subunit [10].

While metformin has been previously shown to decrease cellular ATP levels in hepatocytes owing to its specific inhibition of the mitochondrial respiratory-chain complex 1 [11-13], some of the early works suggested that the drug activates AMPK without increasing the AMP:ATP ratio [14, 15]. Although these initial conclusions were later refuted by the authors [16], the absence of clear data linking the mitochondrial effect of metformin to activation of hepatic AMPK still nurtures confusion. Thus, an alternative AMPindependent hypothesis involving mitochondrial-derived peroxynitrite was recently proposed, prolonging the controversy [17]. Taken together, a clarification of the mechanism by which metformin activates hepatic AMPK is required, especially in humans.

The aim of this study was to test the hypothesis that activation of AMPK by metformin in primary hepatocytes is consecutive to change in cellular energy status owing to metformin's mitochondrial inhibitory effect on the respiratory-chain complex 1 .

\section{Methods}

Materials All chemicals were purchased from SigmaAldrich (St Louis, MO, USA). 
Ethics All experiments performed in animals were done in accordance with the Institute for Laboratory Animal Research Guide for the Care and Use of Laboratory Animals. All procedures on human tissues have received approval from the university and hospital Ethical Review Boards (St Luc Hospital, Brussels, Belgium).

Generation of liver-specific Ampka1/2 knockout mice The generation of liver-specific Ampko1/2 (also known as Prkaal/2) ${ }^{-/}$mice has been described previously [18].

Isolation and primary culture of murine and human hepatocytes For rodent experiments, liver cells were prepared by the collagenase method of Berry and Friend [19], modified by Groen et al. [20], from male Wistar rats (200$300 \mathrm{~g}$ ) or from male mice (25-30 g) after anaesthesia with sodium pentobarbital (6 mg/100 g body weight) or ketamin/ xylazin (8/1 mg/100 g body weight), respectively. For human experiments, hepatocytes were isolated from whole livers or liver segments not used for transplantation, using collagenase P (Roche, Mijdrecht, the Netherlands). For primary culture, rat or human hepatocytes were first seeded for 3 to $4 \mathrm{~h}$ on type I collagen-coated dishes $\left(2 \times 10^{4}\right.$ cells/ $\mathrm{cm}^{2}$ ) and then cultured in M199 medium (Invitrogen, Leek, the Netherlands) supplemented with antibiotics in the presence of the indicated concentrations of metformin.

Western blot analysis Hepatocytes or liver samples were lysed in ice-cold buffer containing: $50 \mathrm{mmol} / \mathrm{l}$ HEPES (pH 7.6), $50 \mathrm{mmol} / \mathrm{l} \mathrm{NaF}, 50 \mathrm{mmol} / \mathrm{l} \mathrm{KCl}, 5 \mathrm{mmol} / \mathrm{l} \mathrm{NaPPi}$, $1 \mathrm{mmol} / 1$ EDTA, $1 \mathrm{mmol} / 1$ EGTA, $1 \mathrm{mmol} / 1$ dithiothreitol, $5 \mathrm{mmol} / 1 \beta$-glycerophosphate, $1 \mathrm{mmol} / 1$ sodium vanadate, $1 \%$ NP40 (vol./vol.) and protease inhibitors cocktail (Complete; Roche). Homogenates were centrifuged $\left(16,000 \mathrm{~g} ; 15 \mathrm{~min}, 4^{\circ} \mathrm{C}\right)$ and the protein content of the supernatant fraction was determined using a kit (BCA Protein Assay Kit; Pierce, Rockford, IL, USA). Proteins (10-50 $\mu \mathrm{g})$ were separated by $7-10 \%$ SDS-PAGE, followed by transfer to a polyvinylidene fluoride transfer membrane. Membranes were blocked for $1 \mathrm{~h}$ at room temperature in TRIS-buffered saline Tween-20 buffer with 5\% non-fat dry milk, followed by overnight incubation with phosphospecific or total antibodies (all of them cross-reacting with human, rat and mouse forms, see electronic supplementary material [ESM] Table 1). Blots were then incubated for $1 \mathrm{~h}$ with horseradish peroxidase-conjugated secondary antibodies at room temperature. Bands were visualised by enhanced chemiluminescence and quantified using Image $\mathbf{J}$ (NIH, Bethesda, MD, USA).

AMPK assays AMPK activity was assayed either after precipitation with $10 \%$ (wt/vol.) polyethylene glycol 6000 or after immunoprecipitation with specific antibodies directed against $\alpha 1-$ or $\alpha 2-A M P K$ catalytic subunits (Kinasource, Dundee, UK), as described [18].

Determination of adenine nucleotide concentrations Samples of the cell suspension or cultured hepatocytes were lysed in ice-cold $\mathrm{HClO}_{4}$-EDTA (5\% wt/vol., $25 \mathrm{mmol} / \mathrm{l}$ ) and centrifuged $(13000 \mathrm{~g}, 2 \mathrm{~min})$. The supernatant fractions were immediately neutralised and determination of adenine nucleotides was performed by high-performance liquid chromatography, as previously described [18].

$R N A$ purification and quantitative reverse transcriptioncoupled real-time PCR RNA was extracted from isolated hepatocytes using an RNA isolation reagent (Tripure; Roche). Total RNA (2 $\mu \mathrm{g})$ was reverse-transcribed and quantitative real-time PCR then performed with a kit (SYBR Green Core; Bio-Rad, Veenendaal, the Netherlands) on a thermal cycler (MyIQ; Bio-Rad). mRNA expression was normalised to GAPDH mRNA content and expressed as arbitrary units. All the primer sets used were designed to span an exon (avoiding eventual amplification of gDNA) and have an efficiency of $\sim 100 \pm 5 \%$ (ESM Table 2).

Determination of mitochondrial oxygen consumption rate in intact and permeabilised hepatocytes Mouse or human hepatocytes (7-8 $\mathrm{mg}$ dry cells per $\mathrm{ml}$ ) were incubated in a shaking water bath at $37^{\circ} \mathrm{C}$ in closed vials containing $2 \mathrm{ml}$ Krebs-Ringer bicarbonate-calcium buffer $(120 \mathrm{mmol} / \mathrm{l} \mathrm{NaCl}$, $4.8 \mathrm{mmol} / \mathrm{l} \mathrm{KCl}, 1.2 \mathrm{mmol} / 1 \mathrm{KH}_{2} \mathrm{PO}_{4}, 1.2 \mathrm{mmol} / 1 \mathrm{MgSO}_{4}$, $24 \mathrm{mmol} / 1 \mathrm{NaHCO}_{3}, 1.3 \mathrm{mmol} / 1 \mathrm{CaCl}_{2}, \mathrm{pH}$ 7.4) in equilibrium with a gas phase containing $\mathrm{O}_{2} / \mathrm{CO}_{2}(19: 1)$ and supplemented with lactate/pyruvate/octanoate $(20 / 2 / 4 \mathrm{mmol} /$ 1) in the presence or not of $5 \mathrm{mmol} / \mathrm{l}$ metformin. After $30 \mathrm{~min}$, the cell suspension was saturated again with $\mathrm{O}_{2} / \mathrm{CO}_{2}$ for $1 \mathrm{~min}$ and immediately transferred into a stirred oxygraph chamber equipped with a Clark oxygen electrode (HEITO, Paris, France). The mitochondrial oxygen consumption rate $\left(\mathrm{OO}_{2}\right)$ was measured at $37^{\circ} \mathrm{C}$ before and after successive addition of $0.5 \mu \mathrm{mol} / 1$ oligomycin and $150 \mu \mathrm{mol} / \mathrm{l} 2,4$ dinitrophenol (DNP). To permeabilise hepatocytes, intact cells were first incubated for $30 \mathrm{~min}$ as described above, then collected by centrifugation and resuspended in $\mathrm{KCl}$ medium (125 mmol/1 KCl, 20 mmol/l TRIS-HCl, 1 mmol/1 EGTA and $5 \mathrm{mmol} / \mathrm{l}$ Pi-TRIS, pH 7.2) containing $200 \mu \mathrm{g} / \mathrm{ml}$ digitonin. After $3 \mathrm{~min}$ at $37^{\circ} \mathrm{C}$, the permeabilised hepatocytes were transferred to the oxygraph. As indicated, $5 \mathrm{mmol} / 1$ glutamate-TRIS plus $2.5 \mathrm{mmol} / 1$ malate-TRIS, or $5 \mathrm{mmol} / 1$ succinate-TRIS plus $0.5 \mathrm{mmol} / 1$ malate-TRIS plus $1.25 \mu \mathrm{mol} / 1$ rotenone were added. $\mathrm{JO}_{2}$ was measured before and after the successive addition of $1 \mathrm{mmol} / \mathrm{l}$ ADP-TRIS, $0.5 \mu \mathrm{g} / \mathrm{ml}$ oligomycin and $50 \mu \mathrm{mol} / 1 \mathrm{DNP}$. All the results are expressed in percentage of the maximum activity of cytochrome oxidase, after addition of $0.15 \mu \mathrm{g} / \mathrm{ml}$ antimycin 
and $1 \mathrm{mmol} / 1 \mathrm{~N}, N, N^{\prime}, N^{\prime}$-tetramethyl-1,4-phenylenediamine (TMPD) plus $5 \mathrm{mmol} / \mathrm{l}$ ascorbate to normalise the results according to respective mitochondrial content in wild-type and liver-specific $A m p k \alpha 1 / 2^{-/}$mice.

Statistics All data are expressed as mean \pm SEM. Statistical analysis was performed using SPSS 17.0 software package for Windows (SPSS, Chicago, IL, USA) with two-tailed unpaired Student's test or one-way/two-way ANOVA, followed by a Tukey's post hoc test for multiple comparisons. Differences between groups were considered statistically significant at $p<0.05$.

\section{Results}

Effects of metformin on AMPK activity and cellular energy status in rat hepatocytes In a first set of experiments, the effects of metformin on AMPK activity and AMP:ATP ratio were studied in primary rat hepatocytes. As shown in Fig. 1a, metformin induced a significant time- and dose-
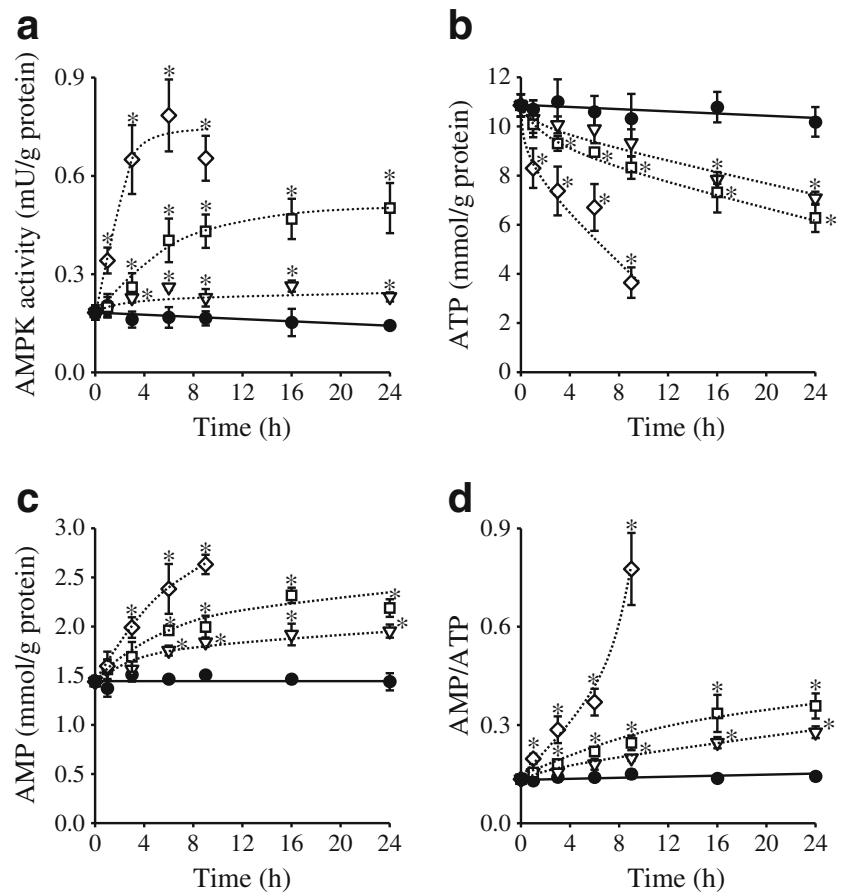

Fig. 1 Time- and dose-dependent effects of metformin on AMPK activity and intracellular adenine nucleotide levels in primary rat hepatocytes. Primary cultured rat hepatocytes were incubated for 0 , $0.5,3,6,9,18$ and $24 \mathrm{~h}$ in M199 medium with different concentrations of metformin (white triangle, $0.5 \mathrm{mmol} / \mathrm{l}$; white square, $1 \mathrm{mmol} / \mathrm{l}$; white diamond, $5 \mathrm{mmol} / \mathrm{l}$ ) or vehicle (black dots). a AMPK activity was measured after polyethylene glycol fractionation. b Intracellular ATP and (c) AMP concentrations were determined by HPLC, and the AMP:ATP ratios (d) calculated. Results are expressed as means \pm SEM; $n=3-4 ;{ }^{*} p<0.05$ compared with control-vehicle dependent activation of AMPK, which was already detectable at $0.5 \mathrm{mmol} / 1$ after $3 \mathrm{~h}$. This dose-dependent effect persisted after $24 \mathrm{~h}$ and was paralleled by a concomitant decrease in ATP and increase in AMP intracellular levels (Fig. 1b, c), resulting in a significant increase of the AMP: ATP ratio (Fig. 1d).

Species-specific activation of AMPK by metformin We next assessed whether the effects of metformin on AMPK activity and the AMP:ATP ratio were similar in rat and human primary hepatocytes. As shown in Fig. 2, metformin increased the AMP:ATP ratio, AMPK activity and Ser79-acetyl-CoA carboxylase (ACC) phosphorylation at concentrations higher than $100 \mu \mathrm{mol} / \mathrm{l}$ in rat and human hepatocytes. However, the extent of AMPK activation by metformin was higher in
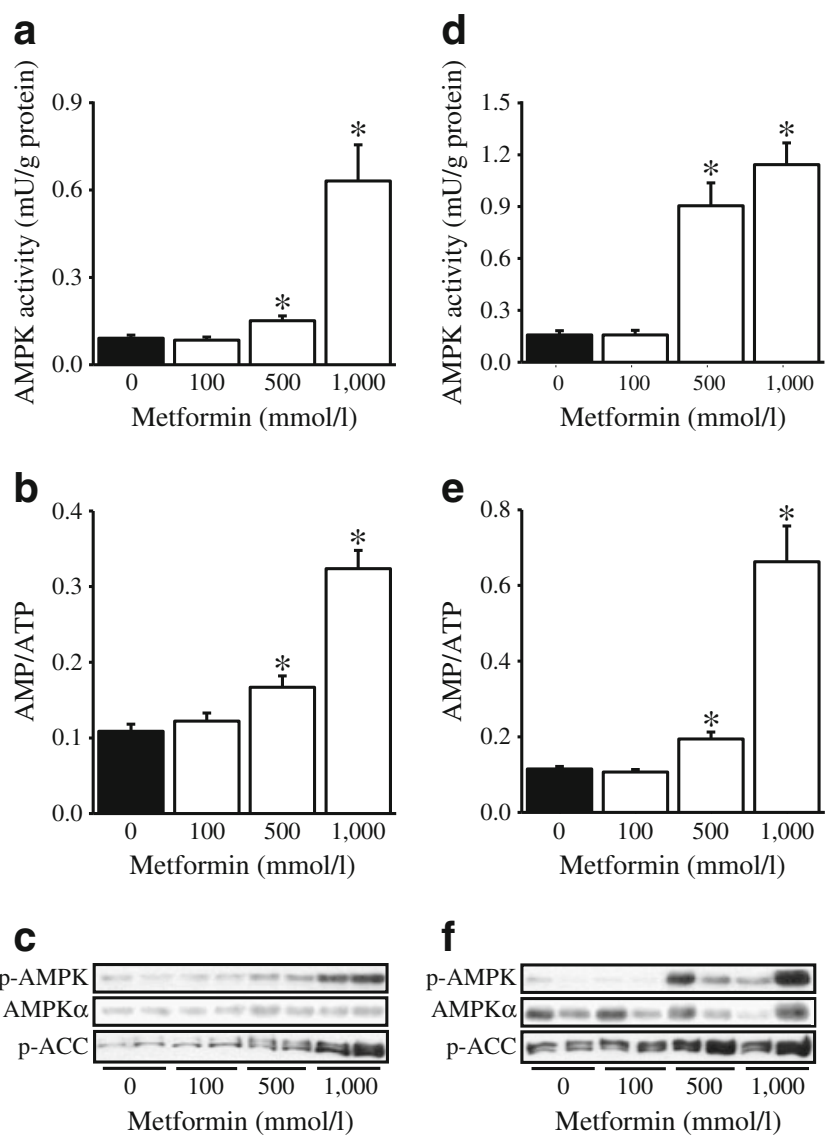

Fig. 2 Dose-dependent effects of metformin on AMPK activity and the AMP:ATP ratio in primary rat and human hepatocytes. a-c Primary cultured hepatocytes isolated from rats or $(\mathbf{d}-\mathbf{f})$ human liver segments were incubated for $24 \mathrm{~h}$ in M199 medium with increasing concentrations of metformin (white bars) or vehicle (black bars). a, d AMPK activity was measured after polyethylene glycol fractionation and the AMP:ATP ratios (b, e) were calculated after determination of intracellular adenine nucleotides by HPLC. c, f The phosphorylation states (p) of Thr172-AMPK and Ser79-ACC, and the abundance of AMPK $\alpha$ were assessed by western blot. Results (a, b, d, e) are expressed as means \pm SEM; $n=3-4 ;{ }^{*} p<0.05$ compared with controlvehicle 
human than in rat hepatocytes at intermediate concentrations of the drug ( $472 \%$ versus $66 \%$ at $500 \mu \mathrm{mol} / 1$, respectively) (Fig. 2a, d), although the increase in the AMP:ATP ratio was similar (69\% versus 54\%, respectively) (Fig. 2b, e), suggesting a species-specific difference in AMPK sensitivity toward AMP, ADP and/or ATP. Strikingly, the determination of isoform-specific AMPK $\alpha$ activities after immunoprecipitation of the $\alpha 1$ or $\alpha 2$ AMPK catalytic subunits revealed that AMPK $\alpha 2$ activity was undetectable in human compared with rat hepatocytes, while AMPK $\alpha 1$ activity was comparable in the basal condition (Fig. 3). Accordingly, metformin only increased AMPK $\alpha 1$ activity in human hepatocytes, although both AMPK $\alpha$ isoforms were activated by the drug in rat hepatocytes. The apparent species-specific difference in AMPK $\alpha 2$ activity was confirmed by the absence of protein and mRNA expression of this catalytic subunit in human hepatocytes (Fig. 4). Interestingly, protein levels and mRNA hepatic expression of the $\beta$ and $\gamma$ regulatory subunits also significantly differed between species, showing that the $\beta 2$, $\gamma 1$ and truncated $\gamma 2$ forms are expressed in human hepatocytes, whereas the $\beta 1, \gamma 1$ and $\gamma 2$ forms are mostly present in rat and mouse hepatocytes (Fig. 4). We confirmed that these species-specific differences in AMPK isoform levels is also found in whole liver (ESM Fig. 1), excluding experimental bias induced by hepatocytes isolation.

Taken together, the fact that AMPK activation by metformin is associated with an elevated AMP:ATP ratio in hepatocytes strongly suggests that inhibition of the mitochondrial machinery by the drug might be the main underlying mechanism for hepatic activation of the kinase.

Mechanism(s) of AMPK activation by metformin To investigate the mechanisms by which metformin affects the cellular energy state, together with the putative involvement of AMPK in this process, we used hepatocytes from wild-
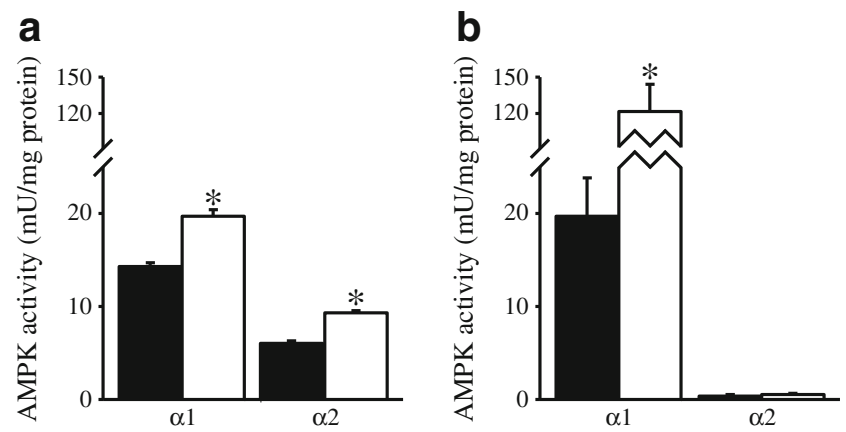

Fig. 3 Effects of metformin on AMPK $\alpha 1$ and $\alpha 2$ activities. a Primary cultured hepatocytes isolated from rats or (b) human liver segments were incubated for $24 \mathrm{~h}$ in M199 medium in the presence of $500 \mu \mathrm{mol} / 1$ metformin (white bars) or vehicle (black bars). AMPK $\alpha 1$ and $\alpha 2$ activity was measured after immunoprecipitation with isoform-specific antibodies. Results are expressed as means \pm SEM; $n=3-4 ; * p<0.05$ compared with control-vehicle type and liver-specific Ampko1/2 ${ }^{-/-}$mice. Freshly isolated hepatocytes were incubated with metformin, and AMPK activity and expression, as well as the AMP:ATP ratio and $\mathrm{JO}_{2}$ were measured. As expected, metformin increased the AMP:ATP ratio, AMPK activity and Ser79-ACC phosphorylation in hepatocytes from wild-type mice (Fig. $5 \mathrm{a}-\mathrm{c}$ ). In hepatocytes from liver-specific Ampk $\alpha 1 / 2^{-/-}$mice, AMPK expression, activity and activation could not be detected (Fig. 5a, b), but the increase in the AMP:ATP ratio induced by metformin was still present and even significantly higher than in hepatocytes from wild-type mice (Fig. 5c). Metformin induced a similar inhibition of $\mathrm{JO}_{2}$ in wild-type and liver-specific $A m p k \alpha 1 / 2^{-/}$mice, an effect that persisted after addition of the mitochondrial oxidative phosphorylation (OXPHOS) uncoupler DNP (Fig. 5d, e). This clearly indicates that the inhibitory effect of metformin on $\mathrm{JO}_{2}$ was exerted on the electron transfer chain rather than on downstream step(s) linked to ATP synthesis. The effect of metformin in intact cells from wild-type and liver-specific Ampk $\alpha 1 / 2^{-/-}$mice was further investigated after permeabilisation of the plasma membrane by digitonin, allowing the mitochondrial OXPHOS pathway to be investigated in situ. In the presence of glutamate/malate, a substrate for the respiratory-chain complex 1 , a significant decrease in mitochondrial respiratory rates could be detected after metformin pre-treatment of cells from wild-type and liverspecific Ampko1/2 $2^{--}$mice, occurring regardless of the mitochondrial energy state (Fig. 5f, g). By contrast, no differences were observed with succinate/malate, a substrate for the respiratory-chain complex 2 (Fig. 5h, i). Importantly, a similar specific inhibition of the mitochondrial respiratory-chain complex 1 by metformin was found in freshly isolated human hepatocytes incubated in the same conditions (Fig. 6).

Collectively, these results demonstrate that the inhibition of $\mathrm{JO}_{2}$ and resultant increase in the AMP:ATP ratio induced by metformin in hepatocytes is due to a specific and AMPK-independent inhibition of the mitochondrial respiratory-chain complex 1 .

\section{Discussion}

Collectively, our results show that activation of AMPK by metformin in primary hepatocytes from rodents and humans is due to a decrease in cellular energy status resulting from metformin's AMPK-independent and specific inhibition of mitochondrial respiratory-chain complex 1 . Strikingly, we report here for the first time that the distribution of AMPK catalytic and regulatory subunits in hepatocytes differs between rodents and humans. This new important finding suggests possible consequences for pharmaceutical strate- 


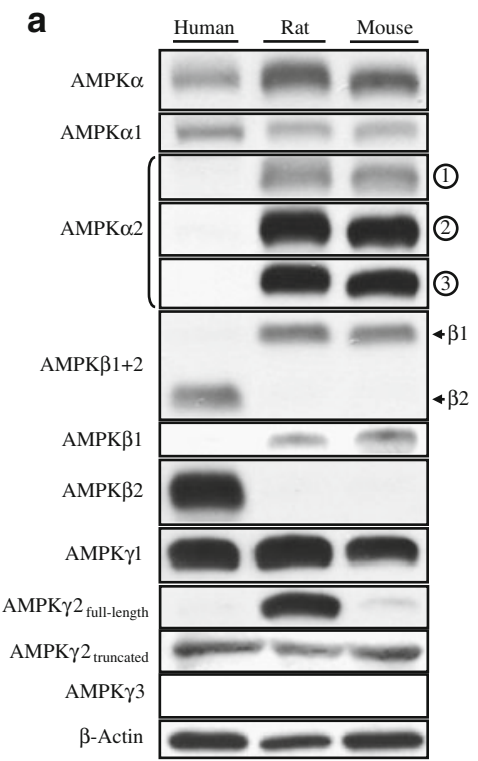

Fig. 4 a Protein levels of AMPK subunits and (b) the corresponding mRNA gene expression were determined in hepatocytes isolated from human (black bars), rat (hatched bars) or mouse (white bars) livers by western blot and RT quantitative PCR, respectively. Western blot analysis (a) is representative of three separate experiments and shows that AMPK subunits migrated to their expected molecular mass

gies focusing on the development of AMPK activators for the treatment of metabolic diseases.

The mitochondrial respiratory-chain complex 1 is the primary target of metformin The inhibition of the mitochondrial respiratory-chain complex 1 by metformin was first reported in perfused livers and isolated rat hepatocytes by Leverve's group [12] and later confirmed in various other cellular models by us [21-25] and others [11, 13, 26, 27]. Due to its weaker lipophilic property, metformin, in contrast to the other biguanides, induces only mild and specific inhibition of the respiratory-chain complex 1 and does not affect OXPHOS downstream machinery (Figs 5 and 6; B. Guigas, unpublished results). In the present manuscript, we show for the first time that this mitochondrial effect of metformin is also present in human hepatocytes, confirming that the respiratory-chain complex 1 constitutes the primary target of the drug, whatever the species.

Although the exact mechanisms by which metformin decreases complex 1 activity remains unknown to date, it has been shown that this effect requires intact cells $[12,22]$ and, at least in hepatocytes, is not prevented by inhibition of nitric oxide synthase or by various reactive oxygen species scavengers [12]. On the other hand, the mechanism by which metformin activates AMPK is still being discussed. Despite the indisputable mitochondrial effect of

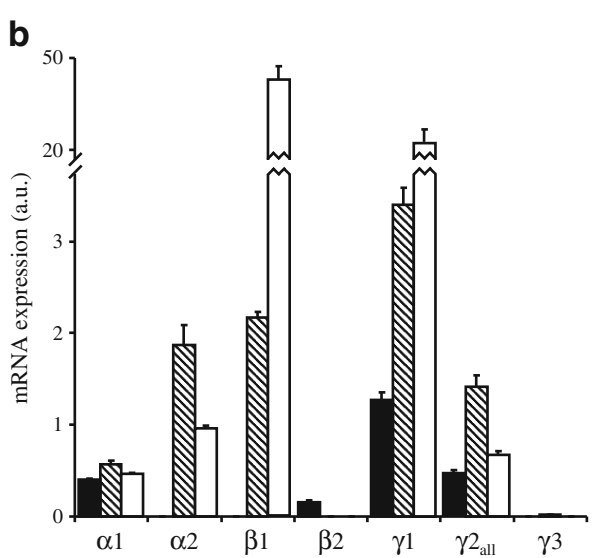

$(\alpha 1,62 \mathrm{kDa} ; \alpha 2,62 \mathrm{kDa} ; \beta 1,38 \mathrm{kDa} ; \beta 2,30 \mathrm{kDa} ; \gamma 1,38 \mathrm{kDa}$; $\left.\gamma 2_{\text {full-length }}, \sim 75 \mathrm{kDa} ; \gamma 2_{\text {truncated, }} \sim 55 \mathrm{kDa} ; \gamma 3,55 \mathrm{kDa}\right)$. The western blots for $\mathrm{AMPK} \alpha 2$ were performed using three different primary antibodies cross-reacting with human, rat and mouse forms (see ESM Table 1). b The results from RT quantitative PCR were corrected for $G A P D H$ and are expressed as means $\pm \operatorname{SEM}(n=3)$. a.u., arbitrary units

the drug, Fryer et al. and Hawley et al. initially reported that metformin activated AMPK in muscle and rat hepatoma cells without affecting the AMP:ATP ratio $[14,15]$. In the present study, we clearly demonstrate that the activation of AMPK by metformin is associated with an increased AMP: ATP ratio resulting from inhibition of the respiratory-chain complex 1. These discrepancies may be explained by technical differences in nucleotide measurement [28] and subcellular AMP compartmentalisation [29], or by the fact that immortalised cell lines are highly glycolytic and therefore much less sensitive to impairment of mitochondrial OXPHOS for ATP supply [30]. Interestingly, metformin was shown to increase cytosolic AMP and AMPK activity in heart [29]. In addition, the involvement of complex 1 in the activation of AMPK by metformin has also been elegantly demonstrated in pancreatic MIN6 cells, by showing that methyl succinate, a substrate of complex 2 that bypasses the inhibition of complex 1 by the drug, prevented AMPK activation [26]. Finally, two recent publications also support the finding that metformin activates AMPK via changes in cellular energy status. Thus Hawley et al. showed that the activation of AMPK by metformin is abolished in a cell line stably expressing AMPK complexes that contain an AMP-insensitive $\gamma 2$ mutant; this indicates that increased cytosolic AMP triggers activation of the kinase by the drug [28]. On the other hand, Foretz et al. also demonstrated that, contrary to what was 


\section{a}

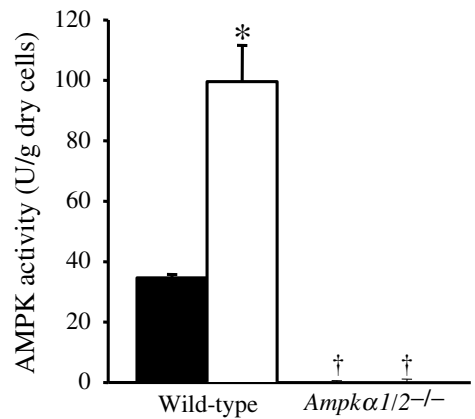

b

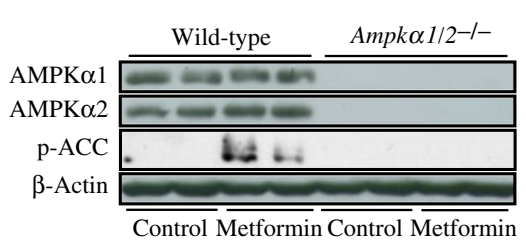

C

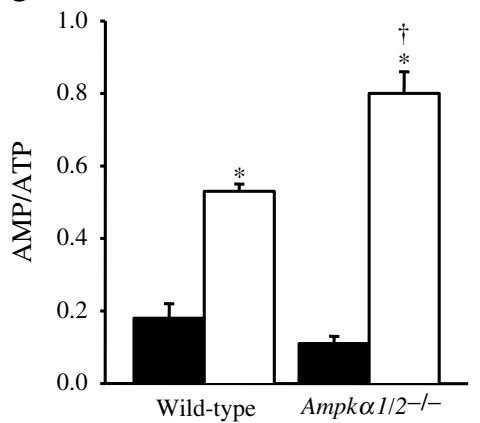

d

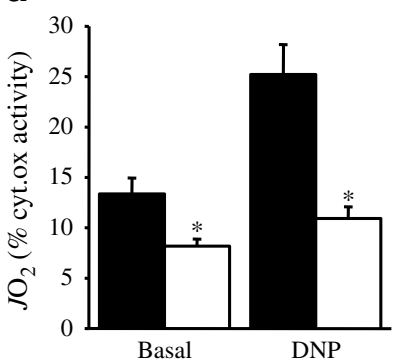

f

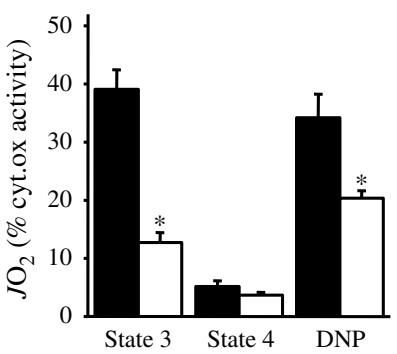

h

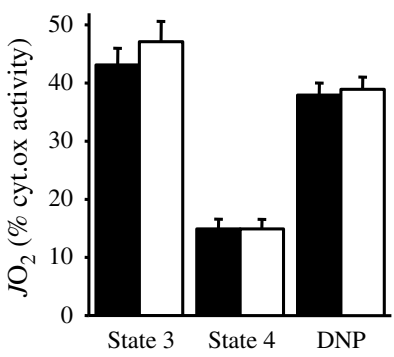

e

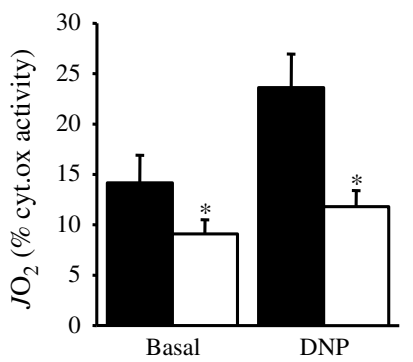

g

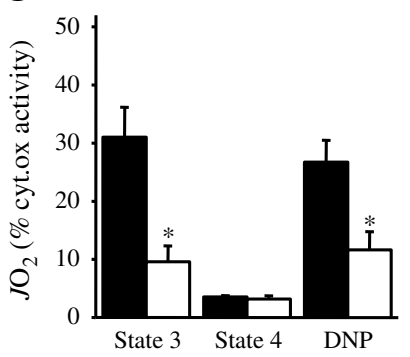

i

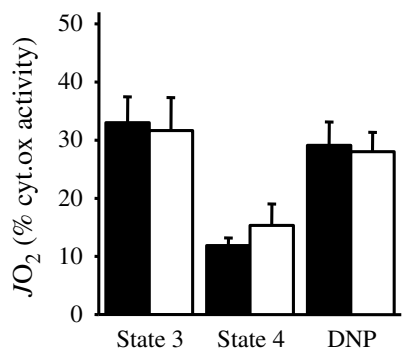

Fig. 5 Effects of metformin on AMPK activity, AMP:ATP ratio, and cellular and mitochondrial $\mathrm{JO}_{2}$ in intact or permeabilised hepatocytes from wild-type and liver-specific Ampk $1 / 2^{-/-}$mice. Hepatocytes from wild-type or Ampk $\alpha 1 / 2^{-/-}$mice were incubated at $37^{\circ} \mathrm{C}$ in a Krebs/bicarbonate medium containing lactate/pyruvate/octanoate $(20$, $2,4 \mathrm{mmol} / \mathrm{l}$ ) in the presence of vehicle (black bars) or $5 \mathrm{mmol} / \mathrm{l}$ metformin (white bars). After $30 \mathrm{~min}$ incubation, AMPK activity in $\mathrm{U} / \mathrm{g}$ of dry cells (a), isoform-specific AMPK $\alpha$ abundance and Ser79-ACC phosphorylation (p) state (b), and the AMP:ATP ratio (c) were determined. d-i Oligomycin-sensitive $\mathrm{JO}_{2}$ was measured after the successive addition of $6 \mu \mathrm{g} / \mathrm{ml}$ oligomycin (basal) and $100 \mu \mathrm{mol} / 1 \mathrm{DNP}$ in separate experiments, after hepatocytes from wild-type $(\mathbf{d}, \mathbf{f}, \mathbf{h})$ and liver-specific $A m p k \alpha 1 / 2^{-/}$(e, g, i) mice had been incubated as described above and permeabilised in a $\mathrm{KCl}$ medium containing $200 \mu \mathrm{g} / \mathrm{ml}$ digitonine. $J \mathrm{O}_{2}$ was measured in the presence of glutamate/malate $(5 \mathrm{mmol} / \mathrm{l} ; 2.5 \mathrm{mmol} / \mathrm{l})(\mathbf{f}, \mathbf{g})$ or succinate/malate/ rotenone $(5 \mathrm{mmol} / \mathrm{l} ; 0.5 \mathrm{mmol} / \mathrm{l} ; 1.25 \mu \mathrm{mol} / \mathrm{l})(\mathbf{h}, \mathbf{i})$ after the successive addition of $1 \mathrm{mmol} / \mathrm{l} \mathrm{ADP}$ (state 3), $6 \mu \mathrm{g} / \mathrm{ml}$ oligomycin (state 4 ) and $75 \mu \mathrm{mol} / 1 \mathrm{DNP}$. All $\mathrm{JO}_{2}$ results are expressed as percentage of the maximum activity of cytochrome oxidase (cyt.ox) determined after the final addition of $0.15 \mu \mathrm{g} / \mathrm{ml}$ antimycin and TMPD/ascorbate $(1 \mathrm{mmol} / \mathrm{l}$, $5 \mathrm{mmol} / \mathrm{l})$. Results are expressed as means \pm SEM; $n=3 ;{ }^{*} p<0.05$ compared with control-vehicle; ${ }^{\dagger} p<0.05$ compared with wild-type mice

activity [32]. To rule out the possibility that the mitochondrial effect of metformin is secondary to AMPK activation via some putative AMP-independent mechanism, we used AMPK-null hepatocytes isolated from liver-specific Ampko1/2 $2^{--}$mice [18, 30]. By showing that metformin inhibited the respiratory-chain complex 1 in hepatocytes from wild-type and liver-specific Ampk $\alpha 1 / 2^{-/-}$mice, we can clearly rule out the notion that a primary effect of the drug is mediated by AMPK through phosphorylation of some subunits of the respiratory-chain complex 1 and/or other protein kinases, thus affecting respiratory-chain complex 1 

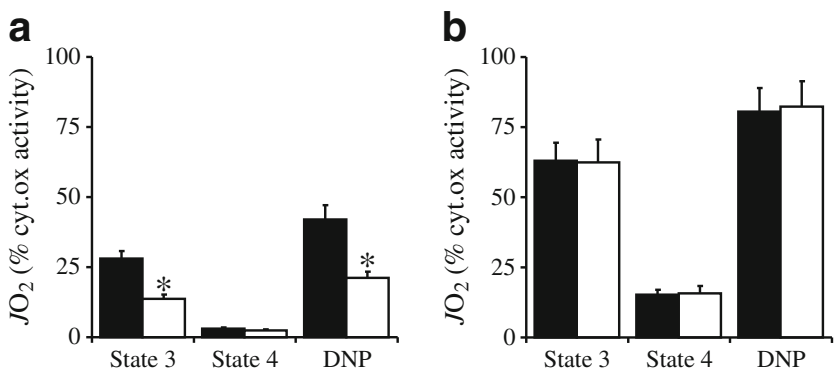

Fig. 6 Effects of metformin on $\mathrm{JO}_{2}$ in permeabilised human hepatocytes. Hepatocytes isolated from human liver segments were incubated in the presence of vehicle (black bars) or $5 \mathrm{mmol} / \mathrm{l}$ metformin (white bars), and the $\mathrm{JO}_{2}$ was measured in the presence of (a) glutamate/malate $(5 \mathrm{mmol} / 1,2.5 \mathrm{mmol} / \mathrm{l})$ or (b) succinate/malate/ rotenone $(5 \mathrm{mmol} / \mathrm{l} ; 0.5 \mathrm{mmol} / \mathrm{l} ; 1.25 \mu \mathrm{mol} / \mathrm{l})$ as described (Fig. 5). The results are expressed as means \pm SEM; $n=3 ; * p<0.05$ compared with control-vehicle

mitochondrial proteins regulating OXPHOS. The more pronounced effect of metformin on the AMP:ATP ratio in primary hepatocytes from liver-specific Ampko1/2 ${ }^{-/}$compared with wild-type mice (Fig. 5) could be explained by a decrease in ATP synthesis secondary to impaired mitochondrial biogenesis and/or by a lack of AMPK-mediated inhibition of ATP consumption, as previously reported $[18,30]$.

Species-specific differences in hepatic abundance of AMPK subunits The AMPK protein is a heterotrimer composed of a catalytic $\alpha$ subunit and two regulatory $\beta$ and $\gamma$ subunits, in a ratio of $1 \alpha: 1 \beta: 1 \gamma$, all of which are required for the formation of a stable and fully functional AMPK complex $[8,9]$. While some minor differences could be shown, depending on the fibre types, for the $\gamma$ regulatory subunit [33], it is well established that the $\alpha 1$ and $\alpha 2$ catalytic subunits are both expressed in human and rodent skeletal muscle, and that the $\alpha 2 \beta 2 \gamma 1$ complex constitutes the majority of AMPK heterotrimers in this metabolic tissue, showing a high degree of consistency between species [33-37]. In the present study, the mRNA expression pattern and protein levels of the AMPK subunits found in rodent livers is in agreement with those previously reported $[35,38]$, leading to many possible combinations of heterotrimers containing either $\alpha 1$ or $\alpha 2$ catalytic subunits, and $\beta 1, \gamma 1$ and $\gamma 2$ regulatory subunits. As far as we know, our finding showing that the abundance of hepatic AMPK subunits differs in humans compared with rodents is unprecedented. Thus, it might be speculated that the resulting differences in heterotrimeric composition of AMPK could affect the regulation of kinase activity, notably its sensitivity toward AMP. Indeed, it is striking that the activation of AMPK by metformin seemed more potent, although the increase of the AMP:ATP ratio in humans compared with rat primary hepatocytes was similar (Fig. 2), suggesting that the human AMPK $\alpha 1 \beta 2 \gamma 1$ or $\alpha 1 \beta 2 \gamma 2$ truncated complexes could be more sensitive to subtle changes in cellular energy status. However, this is in contradiction with previous in vitro results showing that, in rat liver extract, AMPK complexes containing the $\alpha 2 / \beta 2$ isoforms had a greater dependence on AMP [35, 39]. Interestingly, it has been very recently shown that ADP, like AMP, could also bind to the $\gamma$ subunit, leading to modulation of the AMP-triggered phosphorylation of AMPK on Thr172 [10]. It seems therefore possible that subtle species-specific differences in the metformin-induced increase of the ADP: ATP ratio could also be involved. Unfortunately, the determination of intracellular ADP levels by HPLC was technically not possible in our conditions, so further investigations are required to clarify this point. It is worth mentioning that AMPK has also been reported to have a differential and tissue-specific localisation pattern in mammalian cells, with the AMPK $\alpha 1$ subunit being mainly localised in the cytosol, and the AMPK $\alpha 2$ and $\beta 2$ subunits being localised to the nucleus and cytosolic fractions [39-42]. While not as yet clarified, the subcellular localisation of the kinase might have an important functional role, such as regulation of gene expression by phosphorylation of nuclear targets of AMPK. This would therefore suggest that differences in the composition of AMPK complexes between rodents and human hepatocytes could result in different physiological outcomes, including putative nuclear regulatory functions.

Taken together, the species-specific differences shown in AMPK $\alpha \beta \gamma$ complexes in the liver imply that pharmaceutical activation of hepatic AMPK could have different effects in rodents and humans.

Therapeutic relevance of the metformin concentrations used in vitro As shown in Fig. 1, the key determinant of AMPK activation by metformin is a balance between the concentration and the time of exposure to the drug. It is worth noting that most of the in vitro experiments reporting activation of AMPK by metformin were generally performed with drug concentrations far above those found in tissues from rodents after oral administration of metformin [43, 44]. In the present study, we show that hepatic AMPK is activated by metformin at concentrations higher than $100 \mu \mathrm{mol} / \mathrm{l}$, i.e. about five times the highest plasma level reported in humans after a single oral drug administration $[45,46]$. However, the liver is one of the few organs that can accumulate significant amounts of metformin, with tissue concentrations that reached hundreds of $\mu \mathrm{mol} / 1$ in the periportal area $[43,44]$. In addition, the intracellular transport of metformin is mediated by the organic cation transporter 1 (OCT1) [47], the deletion of which in hepatocytes resulted in impairment of metformin-induced AMPK activation [48]. Interestingly, increased OCT1 levels and metformin concentrations were recently reported in the liver of mice on a highfat diet [49], suggesting that hepatic accumulation of the drug could be even higher in diabetic patients. 


\section{Conclusions}

We have demonstrated here that the primary target of metformin in rodent and human hepatocytes is the mitochondrial machinery. The specific inhibition of the respiratory-chain complex 1 by the drug leads to a doseand time-dependent decrease in ATP levels, which results in a concomitant increase in cytosolic AMP concentrations (and the AMP:ATP ratio), triggering the activation of AMPK. One of the important new findings of this study is the species-specific differences in the profile of AMPK subunits, suggesting that regulation of the kinase, which is well characterised in rodents, could differ significantly in humans. Further investigations are still required, but this point should certainly be considered when developing new pharmacological agents that target AMPK.

Acknowledgements The authors are grateful to L. Maisin and M. de Cloedt for technical assistance. This work was supported by FNRS, the Inter University Pole of Attraction, the Association pour l'Etude des Diabètes et des Maladies Métaboliques (ALFEDIAM), Agence Nationale de la Recherche (ANR-10-BLAN-1123-01) and the European Union FP6 programme (Exgenesis/LSHM-CT-2004-005272). B. Guigas was recipient of the ICP "Michel de Visscher" Fellowship.

Contribution statement XS, NT and GCvdZ performed experiments, analysed data, and critically reviewed the manuscript; MF and $\mathrm{BV}$ provided mouse model and reagents, contributed to discussion and critically reviewed the manuscript; ES and LH contributed to discussion and critically reviewed the manuscript; BG conceptualised the project, performed experiments, analysed data, wrote and edited the manuscript. All authors approved the final version of the manuscript.

Duality of interest The authors declare that there is no duality of interest associated with this manuscript.

Open Access This article is distributed under the terms of the Creative Commons Attribution Noncommercial License which permits any noncommercial use, distribution, and reproduction in any medium, provided the original author(s) and source are credited.

\section{References}

1. Goodarzi MO, Bryer-Ash M (2005) Metformin revisited: reevaluation of its properties and role in the pharmacopoeia of modern antidiabetic agents. Diabetes Obes Metab 7:654-665

2. Nathan DM, Buse JB, Davidson MB et al (2009) Medical management of hyperglycaemia in type 2 diabetes mellitus: a consensus algorithm for the initiation and adjustment of therapy: a consensus statement from the American Diabetes Association and the European Association for the Study of Diabetes. Diabetologia 52:17-30

3. Natali A, Ferrannini E (2006) Effects of metformin and thiazolidinediones on suppression of hepatic glucose production and stimulation of glucose uptake in type 2 diabetes: a systematic review. Diabetologia 49:434-441
4. UKPDS (1998) Effect of intensive blood-glucose control with metformin on complications in overweight patients with type 2 diabetes (UKPDS 34). UK Prospective Diabetes Study (UKPDS) Group. Lancet 352:854-865

5. Ben Sahra I, Le Marchand-Brustel Y, Tanti JF, Bost F (2010) Metformin in cancer therapy: a new perspective for an old antidiabetic drug? Mol Cancer Ther 9:1092-1099

6. Zhou G, Myers R, Li Y et al (2001) Role of AMP-activated protein kinase in mechanism of metformin action. J Clin Invest 108:1167-1174

7. Shaw RJ, Lamia KA, Vasquez D et al (2005) The kinase LKB1 mediates glucose homeostasis in liver and therapeutic effects of metformin. Science 310:1642-1646

8. Steinberg GR, Kemp BE (2009) AMPK in health and disease. Physiol Rev 89:1025-1078

9. Hardie DG (2007) AMP-activated/SNF1 protein kinases: conserved guardians of cellular energy. Nat Rev Mol Cell Biol 8:774-785

10. Oakhill JS, Steel R, Chen ZP et al (2011) AMPK is a direct adenylate charge-regulated protein kinase. Science 332:1433-1435

11. Brunmair B, Staniek K, Gras F et al (2004) Thiazolidinediones, like metformin, inhibit respiratory complex I: a common mechanism contributing to their antidiabetic actions? Diabetes 53:1052-1059

12. El-Mir MY, Nogueira V, Fontaine E, Averet N, Rigoulet M, Leverve X (2000) Dimethylbiguanide inhibits cell respiration via an indirect effect targeted on the respiratory chain complex I. J Biol Chem 275:223-228

13. Owen MR, Doran E, Halestrap AP (2000) Evidence that metformin exerts its anti-diabetic effects through inhibition of complex 1 of the mitochondrial respiratory chain. Biochem J 348:607-614

14. Fryer LG, Parbu-Patel A, Carling D (2002) The anti-diabetic drugs rosiglitazone and metformin stimulate AMP-activated protein kinase through distinct signaling pathways. J Biol Chem 277:25226-25232

15. Hawley SA, Gadalla AE, Olsen GS, Hardie DG (2002) The antidiabetic drug metformin activates the AMP-activated protein kinase cascade via an adenine nucleotide-independent mechanism. Diabetes 51:2420-2425

16. Hardie DG (2006) Neither LKB1 nor AMPK are the direct targets of metformin. Gastroenterology 131:973

17. Fujita Y, Hosokawa M, Fujimoto S et al (2010) Metformin suppresses hepatic gluconeogenesis and lowers fasting blood glucose levels through reactive nitrogen species in mice. Diabetologia 53:1472-1481

18. Guigas B, Bertrand L, Taleux N et al (2006) 5-Aminoimidazole-4carboxamide-1-beta-D-ribofuranoside and metformin inhibit hepatic glucose phosphorylation by an AMP-activated protein kinaseindependent effect on glucokinase translocation. Diabetes 55:865874

19. Berry MN, Friend DS (1969) High-yield preparation of isolated rat liver parenchymal cells: a biochemical and fine structural study. J Cell Biol 43:506-520

20. Groen AK, Sips HJ, Vervoorn RC, Tager JM (1982) Intracellular compartmentation and control of alanine metabolism in rat liver parenchymal cells. Eur J Biochem 122:87-93

21. Detaille D, Guigas B, Chauvin C et al (2005) Metformin prevents high-glucose-induced endothelial cell death through a mitochondrial permeability transition-dependent process. Diabetes 54:2179-2187

22. Detaille D, Guigas B, Leverve X, Wiernsperger N, Devos P (2002) Obligatory role of membrane events in the regulatory effect of metformin on the respiratory chain function. Biochem Pharmacol 63:1259-1272

23. El-Mir MY, Detaille D, R-Villanueva G et al (2008) Neuroprotective role of antidiabetic drug metformin against apoptotic cell death in primary cortical neurons. J Mol Neurosci 34:77-87

24. Guigas B, Detaille D, Chauvin C et al (2004) Metformin inhibits mitochondrial permeability transition and cell death: a pharmacological in vitro study. Biochem J 382:877-884 
25. Batandier C, Guigas B, Detaille D et al (2006) The ROS production induced by a reverse-electron flux at respiratory-chain complex 1 is hampered by metformin. J Bioenerg Biomembr 38:33-42

26. Hinke SA, Martens GA, Cai Y et al (2007) Methyl succinate antagonises biguanide-induced AMPK-activation and death of pancreatic beta-cells through restoration of mitochondrial electron transfer. Br J Pharmacol 150:1031-1043

27. Turner N, Li JY, Gosby A et al (2008) Berberine and its more biologically available derivative, dihydroberberine, inhibit mitochondrial respiratory complex I: a mechanism for the action of berberine to activate AMP-activated protein kinase and improve insulin action. Diabetes 57:1414-1418

28. Hawley SA, Ross FA, Chevtzoff C et al (2010) Use of cells expressing gamma subunit variants to identify diverse mechanisms of AMPK activation. Cell Metab 11:554-565

29. Zhang L, He H, Balschi JA (2007) Metformin and phenformin activate AMP-activated protein kinase in the heart by increasing cytosolic AMP concentration. Am J Physiol Heart Circ Physiol 293:H457-H466

30. Guigas B, Taleux N, Foretz M et al (2007) AMP-activated protein kinase-independent inhibition of hepatic mitochondrial oxidative phosphorylation by AICA riboside. Biochem J 404:499-507

31. Foretz M, Hebrard S, Leclerc J et al (2010) Metformin inhibits hepatic gluconeogenesis in mice independently of the LKB1/AMPK pathway via a decrease in hepatic energy state. J Clin Invest 120:2355-2369

32. Papa S, de Rasmo D, Scacco S et al (2008) Mammalian complex I: a regulable and vulnerable pacemaker in mitochondrial respiratory function. Biochim Biophys Acta 1777:719-728

33. Mahlapuu M, Johansson C, Lindgren K et al (2004) Expression profiling of the gamma-subunit isoforms of AMP-activated protein kinase suggests a major role for gamma3 in white skeletal muscle. Am J Physiol Endocrinol Metab 286:E194-E200

34. Wojtaszewski JF, Birk JB, Frosig C, Holten M, Pilegaard H, Dela F (2005) 5'AMP activated protein kinase expression in human skeletal muscle: effects of strength training and type 2 diabetes. $J$ Physiol 564:563-573

35. Cheung PC, Salt IP, Davies SP, Hardie DG, Carling D (2000) Characterization of AMP-activated protein kinase gamma-subunit isoforms and their role in AMP binding. Biochem J 346:659-669

36. Birk JB, Wojtaszewski JF (2006) Predominant alpha2/beta2/ gamma3 AMPK activation during exercise in human skeletal muscle. J Physiol 577:1021-1032

37. Treebak JT, Glund S, Deshmukh A et al (2006) AMPKmediated AS160 phosphorylation in skeletal muscle is depen- dent on AMPK catalytic and regulatory subunits. Diabetes 55:2051-2058

38. Quinn JM, Tam S, Sims NA et al (2010) Germline deletion of AMP-activated protein kinase beta subunits reduces bone mass without altering osteoclast differentiation or function. FASEB J $24: 275-285$

39. Salt I, Celler JW, Hawley SA et al (1998) AMP-activated protein kinase: greater AMP dependence, and preferential nuclear localization, of complexes containing the alpha2 isoform. Biochem J 334:177-187

40. Kazgan N, Williams T, Forsberg LJ, Brenman JE (2010) Identification of a nuclear export signal in the catalytic subunit of AMP-activated protein kinase. Mol Biol Cell 21: $3433-3442$

41. Kodiha M, Rassi JG, Brown CM, Stochaj U (2007) Localization of AMP kinase is regulated by stress, cell density, and signaling through the MEK $\rightarrow$ ERK1/2 pathway. Am J Physiol Cell Physiol 293:C1427-C1436

42. Suzuki A, Okamoto S, Lee S, Saito K, Shiuchi T, Minokoshi Y (2007) Leptin stimulates fatty acid oxidation and peroxisome proliferator-activated receptor alpha gene expression in mouse $\mathrm{C} 2 \mathrm{C} 12$ myoblasts by changing the subcellular localization of the alpha2 form of AMP-activated protein kinase. Mol Cell Biol 27:4317-4327

43. Wilcock C, Bailey CJ (1994) Accumulation of metformin by tissues of the normal and diabetic mouse. Xenobiotica 24:49-57

44. Wilcock C, Wyre ND, Bailey CJ (1991) Subcellular distribution of metformin in rat liver. J Pharm Pharmacol 43:442-444

45. Tucker GT, Casey C, Phillips PJ, Connor H, Ward JD, Woods HF (1981) Metformin kinetics in healthy subjects and in patients with diabetes mellitus. Br J Clin Pharmacol 12:235-246

46. Graham GG, Punt J, Arora M et al (2011) Clinical pharmacokinetics of metformin. Clin Pharmacokinet 50:81-98

47. Wang DS, Jonker JW, Kato Y, Kusuhara H, Schinkel AH, Sugiyama Y (2002) Involvement of organic cation transporter 1 in hepatic and intestinal distribution of metformin. J Pharmacol Exp Ther 302:510-515

48. Shu Y, Sheardown SA, Brown C et al (2007) Effect of genetic variation in the organic cation transporter 1 (OCT1) on metformin action. J Clin Invest 117:1422-1431

49. Jang EH, Kim HK, Park CS, Kang JH (2010) Increased expression of hepatic organic cation transporter 1 and hepatic distribution of metformin in high-fat diet-induced obese mice. Drug Metab Pharmacokinet 25:392-397 